\title{
Using Genetic Algorithm Image Retrieval Based on Multi- Feature Similarity Score Fusion
}

\author{
Asst Prof P.Kishore Kumar, M.Tech,* Asst Prof M.Radhika, M.Tech,** \\ M.Jyothirmai, (M.Tech), *** \\ *(Department of Electronics \& Communication Engineering, Ravindra engineering college for women, \\ Kurnool) \\ ** (Department of Electronics \& Communication Engineering, GPCET, Kurnool) \\ *** (Department of Electronics \& Communication Engineering, GPCET, and Kurnool)
}

\begin{abstract}
This paper proposes an image retrieval method based on multi-feature similarity score fusion using genetic algorithm. Single feature describes image content only from one point of view, which has a certain one-sided. Fusing multi- feature similarity score is expected to improve the system's retrieval performance. In this paper, the retrieval results from color feature and texture feature are analyzed, and the method of fusing multi-feature similarity score is described. For the purpose of assigning the fusion weights of multi-feature similarity scores reasonably, the genetic algorithm is applied. For comparison, other three methods are implemented. They are image retrieval based on color feature, texture feature and fusion of colortexture feature similarity score with equal weights. The experimental results show that the proposed method is superior to other methods.
\end{abstract}

Keywords: image retrieval; fusion; genetic algorithm

\section{Introduction}

With the rapid development of multimedia and network technology, people can access a large number of multimedia information. For people who want to make full use of multimedia information resources, the primary question is how to query the multimedia information of interest. Text query can be applied to multimedia information retrieval, but it has inherent deficiencies. One hand, text annotation of multimedia information will spend a lot of manpower and resources and it is inefficient. On the other hand, annotated text is usually a person's perception of multimedia information. It is subject to impact of individual difference and state of human and environment, and the described results may be more one-sided. In addition, it is clearly incomplete to describe content-rich multimedia information with a small amount of text. Content Based Image Retrieval (CBIR) techniques appeared in 1990s [1]. It solves the above problems well. It uses low-level features like color, texture and shape to describe image content, and breaks through the limitation of traditional text query technique.

CBIR system can be implemented based on single feature. Single image feature describes the content of an image from a specific angle. It may be suitable for some images, but it also may be difficult to describe other images. Moreover, describing an image with single feature is also incomplete. Representing an image with multi-features from multi-angles is expected to achieve better results. Information is multi- source, and information fusion approach is diverse. The problem how to organize multi-source information in a suitable way to achieve the intended results attracts extensive attention from the researchers in this field.

Information fusion can be carried out in feature level [2]. Information fusion in feature level has advantage in some extent. Because different features reflect the different characteristics of the image, if those features are integrated reasonably, the results will both reserve the discriminate information of multi-feature and eliminate the interference resulted from the difference of multi-feature. B.G. Prasad et al. [3] proposed a technique to retrieve images by region matching using a combined feature index based on color, shape, and location within the framework of MPEG-7. Dominant regions within each image are indexed using integrated color, shape, and location features. Young Deok Chun et al. [4] proposed a content-based image retrieval method based on an efficient combination of multiresolution color and texture features. Color autocorrelograms of the hue and saturation component images in HSV color space are taken as color features. BDIP and BVLC moments of the value component image are adopted as texture features. The color and texture features are extracted in multiresolution wavelet domain and combined. Tai X. Y. et al. [5] defined a new image feature called color-texture correlogram which is the extension of color correlogram. The texture feature extracted by texture spectrum algorithm is combined with color feature vector, and then calculate the spatial correlation of color-texture feature vector. Hui Yu et al. [6] adopted local Fourier transform as a texture representation scheme and derive eight characteristic maps for describing different aspects of co-occurrence relations of image pixels in each channel of color space. Then they calculate the first and second moments of these maps as a representation of the natural color image pixel distribution, resulting in a 48- dimensional feature vector. The novel low-level feature is named color texture 
moments (CTM), which can also be regarded as a certain extension to color moments in eight aspects through eight orthogonal templates.

Information fusion can also be carried out in decision- making level. Image retrievals based on different features are accomplished respectively, and then similarity score came from these results are fused according to some rules, a better result will be returned. Anil K. Jain et al. [7] integrated the results of the shape-based retrieval and the color-based retrieval by combining the associated similarity values with appropriate weights. Xiuqi Li et al. [8] proposed a novel approach to image retrieval using color, texture and spatial information. Color homogram filter, wavelet texture filter, and spatial filter are used in sequence to eliminate images that are dissimilar to a query image in color, texture, and spatial information from the search ranges respectively. The final query ranking is based on the total normalized distance in color, texture, and spatial information of all images passing the three filters. Ilya Markov et al. [9] proposed a technique to combine image similarity measures which takes into account a particular query-image. They introduced mixed-metrics obtained from color and texture metrics by using their weighted linear combination. Mladen Jovic et al. [10] proposed an image similarity method based on the fusion of similarity scores of feature similarity ranking lists. It takes an advantage of combining the similarity value scores of all feature types representing the image content bymeans of different integration algorithms when computing the image similarity.

This paper proposes an image retrieval method based on multi-feature similarity score fusion using genetic algorithm.

This paper analyzed image retrieval results based on color feature and texture feature, and proposed a strategy to fuse multi-feature similarity score. Further, with genetic algorithm, the weights of similarity score are assigned automatically, and a fine image retrieval result is gained.

The rest of this paper is organized as follows. Section describes the extraction method of color feature and texture feature. Section introduced the strategy to fuse multi- feature similarity score. The method assigning weights of multi-feature similarity score by genetic algorithm is given in section . Experimental results and analysis are in section and section concluded the whole paper.

\section{Image Feature Extraction}

The image content is mainly embodied in color, texture and shape etc. The color feature, texture feature and shape feature describe the image content from different angle. More features will provide more information on the image content. This paper focuses on fusion method of multi- feature similarity score. For convenience, this paper only discusses the fusion method of two-feature similarity score. Without loss of generality, the used features are color feature and texture feature. The following part describes the used extraction method of color feature and texture feature.

\section{A. Color featureextraction}

HSV color model forms a uniform color space, which uses a linear gauge. The perceived distance between colors is in proportion to Euclidean distance between corresponding pixels in HSV color model, and conforms to eye's feeling about color. So it is very suitable for color based image similarity comparison. In this paper, the color histogram in HSV color space is taken as the color feature describing image content. For calculating color histogram in HSV color space, HSV color space must first be quantified. According to human cognitive about color, three components of HSV space are quantified in non-uniform manner. Hue is quantized into 16 bins and is among [0, 15]. Saturation is quantized into 4 bins and is among [0,3]. Value is quantized into 4 bins and is among [0,3]. Among those three components, human cognitive about color is mainly based on hue, and then saturation, finally value. So, quantized results are coded as

$C=16 H+4 S+V$

where $C$ is a integer between 0 and 255 . Thus the color feature can be obtained by calculating histogram of an image in HSV space.

\section{B. Texture featurextraction}

In this paper, the statistical properties of image co- occurrence matrix are taken as texture features of an image. Firstly, color image is converted to grayscale image, and the image co-occurrence matrix is gained. Then, the following five statistical properties are calculated to describing image content. They are contrast, energy, entropy, correlation and local stationary. All these statistical properties are calculated in 4 directions, so we can get 20 texture features. At last, we calculated the means and variances of these five kinds of statistical properties, and took the results as the ultimate texture features, denoted as

$T=\left(\mu_{1}, \mu_{2}, \mu_{3}, \mu_{4}, \mu_{5} ; \sigma_{1}, \sigma_{2}, \sigma_{3}, \sigma_{4}, \sigma_{5}\right)$.

\section{Multi-Feature Similarity Score Fusion}

Since the physical meanings of different features are different, and value ranges are totally different, similarity scores of different features can not be compared. So, before multi-feature similarity score are fused, they should be normalized. Similarity scores can be normalized through the following ways. Let $Q$ be the query image. 
By calculating distances between the query image and images in database, similarity score set $\left\{S_{i}\right\}$ can be gotten, where $i=1, \mathbf{L}, N, N$ is the number of images in database. Thus, similarity score normalization can be implemented as

$\underline{S}-\min \{\underline{S}\} \quad=\quad \underline{i} \quad \underline{i}$

$\max \left\{S_{i}\right\}-\min \left\{S_{i}\right\}$

individuals in population $N$ is taken as $I . N$ is set a bigger value, the aim of which is to gain the optindal solution quickly. The individuals are initialized as follows. The solution space is divided into $N$ equal portions, the centers The results of multi-feature similarity scores is

$S=\underline{S}_{\underline{N C i}} \cdot \underline{W}_{\underline{C}}+\underline{S}_{\underline{N T i}} \cdot \underline{W}_{\underline{T}}$,

of which are taken as the initial values of the individuals.

\section{Determination of fitness function}

The fitness of individuals can be evaluated as follows. ${ }^{F i} \quad W+W$

where

$S_{F i c} \quad T$

is the fused similarity score,

$S_{N C i}$

is the

According to the weights $W_{C}$ and $W_{T}$ of $N$ individuals, we can get $N$ groups of image retrieval results. For every group, the top $M$ images are considered. Total number of images is normalized color feature similarity score, $S_{N T i}$ is the

$M N$. By calculating occurrence frequency of images of normalized texture feature similarity score, $W_{C}$ is the weight every group in all images, the fitness of every individual is evaluated. Specific operations are as follows.of color feature similarity score, and $W_{T}$ is the weight of Let $N$ denote if $k$ th image $A$ of $i$ th group $G$ is in $j$ thtexture feature similarity score. By assigning appropriate ${ }^{i k j i} \quad i$ values to $W_{C}$ and $W_{T}$, a fine similarity score fusion can be gained. group $G_{j}$ or not. That can be formulated as

\section{( $1, \mathrm{~A}_{\mathrm{ik}} \in G_{j}$ IV.SIMILARITY SCORE FUSION USING GENETI ALGORITHM}

During the course of similarity score fusion, a key

$N_{i k j}$

$=\{0, \mathrm{~A} \notin G$

(5)problem is how to assign the weights of similarity score. It affects directly the retrieval performance of the system. It can be considered as an optimization problem to assign reasonably the weights of color feature similarity score and texture feature similarity score. That is to find the optimum

Then the occurrence frequency of $k$ th image $A_{i k}$

group $G_{i}$ in all $M N$ images is ${ }_{N}$ of $i$ th in weight value space. So, this problem can be resolved by genetic algorithm. This paper proposed a similarity score fusion method using genetic algorithm. With genetic algorithm the weights of color feature similarity score and texture feature similarity score are assigned optimally.

\section{A. Determination of solutiospace}

The aim of fusing similarity scores is to assign the weights of color feature similarity score and texture feature similarity score to gain a better image retrieval performance. With the consideration of (4), the weight of color feature

$N_{i k}=\sum N_{i k j}$ 
The occurrence frequency of all images of $i$ th group $G_{i}$

in all $M N$ images is

$N_{i}=\sum N_{i k}$.

can be a integer between 0 and $I$,

$$
k=1 \text { similarity score } W_{C}
$$

The normalized version of it iswhere $I$ is a positive integer. Without loss of generality, the weight of texture feature similarity score can be assigned to

$I-W_{C}$. The positive integer $I$ determined the accuracy of

$$
P=
$$

solution. The bigger the value of $I$ is, the higher the accuracy of solution is. But this may take a long time to resolve, and vice versa. To resolve using genetic algorithm,

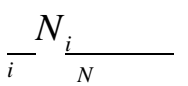

$\sum N_{l}$

$l=0$

(8)the weights should be encoded. The solution should be

expressed as a binary number. So generally the value $I$ is

taken as $2^{L}$, where $L$ is a positive integer, the encoding length of the solution.

\section{B. Population Initialization}

In genetic algorithm, the number of individuals in population and the initial values of the individuals will influence the solution greatly. In this paper, the number of

The bigger $P_{i}$ indicates that the images in $i$ th group $G_{i}$

possess a high proportion in all $M N$ images, and the solution is considered a good one.

In this paper, it is taken as fitness function.

\section{Solving for optimal solution}

The genetic algorithm is implemented in classic mode. The condition for ending the iteration is that the number of iteration is equal to 3 . When the iteration is ended, the maximum $P^{*}=\max P$ is taken as the optimal solution. texture feature poor. Better performance of image retrieval method based on texture feature is expected to increase the According to the optimal solution the weights $W^{*}$ and $W^{*}$ performance of image retrieval method based multi-feature

are assigned, then the image retrieval results with these two weights are taken as the ultimate retrieval results.

\section{Experiments And Analysis}

Corel image library is used to evaluate the proposed algorithm. It contains dozens categories, each of which has about 60 images. The total number of images is about 1000. They are flower, waterfowl, automobile, beach, mountain, loin, bridge, butterfly, plane, building and tiger and so forth. The color feature and texture feature of every image are extracted to build feature database.

Precision rate and recall rate are employed to evaluate the performance of the proposed method. The numbers of returned images are 5, 10, 15, 20, 25, 30, 35, 40, 45 and 50. For comparison, the image retrieval methods based on color feature, texture feature and two-feature similarity score fusion with equal weights are implemented. The precision rate and recall rate relationship of these methods are shown in Fig. 1. 


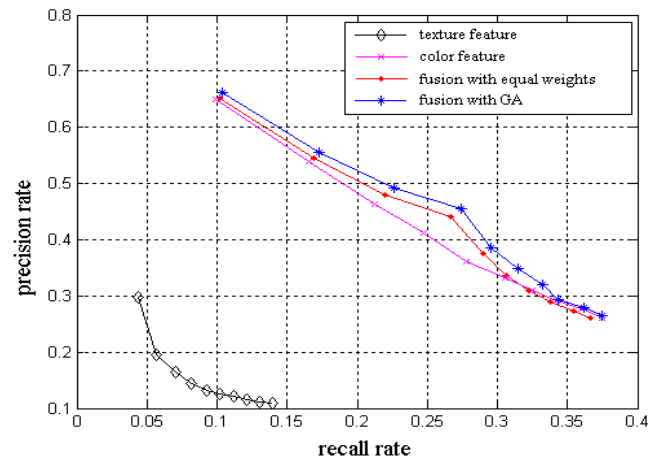

Figure 1. Relationship diagram of precision rate and recall rate.

Fig. 1 showed that image retrieval method based on multi-feature similarity score fusion using genetic algorithm ranked the first. The method with equal weights followed. The method based on color feature is better than the method based on texture feature. The relationship showed in figure 1 reflects mainly the impact of multi-feature similarity score fusion on retrieval results. However, relative to the color- based image retrieval method, performance of these two image retrieval methods based on multi-feature similarity score fusion doesn't increase much. This is mainly due to that compared with image retrieval method based on color feature, the performance of image retrieval method based on texture feature is poor. There are two possible reasons for it. One is that the color difference of different images in this image library is more obvious, and the performance of image retrieval method based on color feature is better. The other is that for this image library, the extracted texture feature may be insufficient to reflect the differences between different classes, which make performance of image retrieval based on similarity score fusion.

\section{Conclusion}

This paper proposed an image retrieval method based multi-feature similarity score fusion. For a query image, multiple similarity score lists based on different features are obtained. Then using genetic algorithm, multifeature similarity scores are fused, and better image retrieval results are gained. In this paper, when we evaluated the fitness of an individual, we considered only the occurrence frequencies of an image in retrieval result, and not the location of an image in retrieval result. However, the location of an image in retrieval result reflects directly the similarity of it and query image. So, this factor should be taken into account when evaluating the fitness of an individual, which is also our future work.

\section{References}

[1] Gudivada V. N., Raghavan V. V., "Content based image retrieval systems," IEEE Computer, vol. 28, pp. 18-22, 1995.

[2] Ritendra Datta, Dhiraj Joshi, Jia Li, James Z. Wang, "Image retrieval: Ideas, influences, and trends of the new age," ACM Computing Surveys, vol. 40, pp. 1-60, 2008.

[3] B.G. Prasad, K.K. Biswas, S.K. Gupta, "Region-based image retrieval using integrated color, shape, and location index," Computer Vision and Image Understanding, vol. 94, pp. 193-233, 2004

[4] Young Deok Chun, Nam Chul Kim, Ick Hoon Jang, "Content-Based Image Retrieval Using Multiresolution Color and Texture Features,” IEEE Transaction on Multimedia, vol. 10, pp. 1073-1084, 2008.

[5] Tai X. Y., Wang L. D. , "Medical Image Retrieval Based on Color- Texture Algorithm and GTI Model, " Bioinformatics and Biomedical Engineering, 2008, ICBBE 2008, The 2nd International Conference on, pp. 2574-2578.

[6] H. Yu, M. Li, H.-J. Zhang, J. Feng, "Color texture moments for content-based image retrieval," In International Conference on Image Processing, pp. 24-28, 2002.

[7] Anil K. Jain , Aditya Vailaya, "Image retrieval using color and shape," Pattern Recognition, vol. 29, pp. 1233-1244, 1996.

[8] Xiuqi Li, Shu-Ching Chen, Mei-Ling Shyu, Borko Furht, "Image Retrieval By Color, Texture, And Spatial Information," In: Proceedings of the the 8th International Conference on Distributed Multimedia Systems (DMS 2002), San Francisco Bay, CA, USA, pp. 152-159, 2002

[9] I. Markov, N. Vassilieva, "Image Retrieval: Color and Texture Combining Based on Query-Image,” ICISP 2008, LNCS 5099, Springer-Verlag Berlin Heidelberg, 2008, pp. 430-438.

[10] M. Jovic, Y. Hatakeyama, F. Dong, K. Hirota, "Image Retrieval Based on Similarity Score Fusion from Feature Similarity

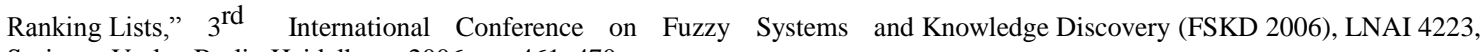
Springer-Verlag Berlin Heidelberg, 2006, pp. 461-470. 